\section{$\beta$ Blocker treatment and other prognostic variables in patients with clinical evidence of heart failure after acute myocardial infarction: evidence from the AIRE study}

\author{
K S Spargias, A S Hall, D C Greenwood, S G Ball
}

Institute for Cardiovascular Research, University of Leeds, Leeds LS2 9JT, UK

K S Spargias A S Hall

S G Ball

Subunit for Medical Statistics, University of Leeds

D C Greenwood

A complete list of the AIRE study administration and collaborators appeared in Lancet 1993;342:821-8.

Correspondence to: Dr Hall.

Accepted for publication 29 July 1998

\begin{abstract}
Objectives-To examine clinical outcomes associated with optional $\beta$ blockade in a population of patients with evidence of heart failure after myocardial infarction. Design and patients-Data from the acute infarction ramipril efficacy (AIRE) study were analysed retrospectively. At baseline $22.3 \%$ of the patients were receiving a $\beta$ blocker. To minimise confounding, $\beta$ blocker and diuretic treatments, presence of clinical signs of heart failure, left ventricular ejection fraction, and 16 other baseline clinical variables were simultaneously entered in a multivariate Cox regression model. In addition, the same analysis was repeated separately within a high and a low risk group of patients, as defined according to the need for diuretic treatment.

Results- $\beta$ Blocker treatment was an independent predictor of reduced risk of total mortality (hazard ratio $0.66,95 \%$ confidence interval (CI) 0.48 to 0.90 ) and progression to severe heart failure $(0.58$, $95 \%$ CI 0.40 to 0.83 ) for the entire study population. There were similar findings in high risk patients requiring diuretics $(0.59,95 \%$ CI 0.40 to 0.86 ; and $0.58,95 \%$ CI 0.38 to 0.89 ).

Conclusions- $\beta$ Blocker treatment is associated with improved outcomes in patients with clinical evidence of mild to moderate heart failure after myocardial infarction. Most importantly, high risk patients with persistent heart failure appear to benefit at least as much as lower risk patients with transient heart failure.

(Heart 1999;81:25-32)
\end{abstract}

Keywords: myocardial infarction; heart failure; left ventricular dysfunction; $\beta$ blockers

Thrombolytic agents, aspirin, and $\beta$ adrenoceptor blockers form the cornerstone of early pharmacological treatment after acute myocardial infarction. ${ }^{1}$ There is overwhelming evidence that administration of $\beta$ blockers decreases short and long term mortality after myocardial infarction and prevents reinfarction and the occurrence of tachyarrhythmias. ${ }^{2-4}$ Since the beneficial effects of $\beta$ blocker treatment were established in postmyocardial infarction trials conducted in the previous decade, there is some uncertainty as to the current value of these agents following routine use of aspirin, thrombolytic, and angiotensin converting enzyme (ACE) inhibitor treatments. ${ }^{15}$

A meta-analysis of studies of long term $\beta$ blocker treatment after myocardial infarction before the routine use of thrombolytic treatment showed a $23 \%$ relative reduction of overall mortality and a $27 \%$ reduction in the rate of reinfarction when these drugs were prescribed. $^{2}$ Despite these persuasive data, many studies of current practice indicate that $\beta$ blockers are inadequately used ${ }^{6-9}$ Recent studies of $\beta$ blockade after myocardial infarction indicate that only $48 \%$ to $58 \%$ of patients who have no contraindications to their use actually receive $\beta$ blockers. ${ }^{78}$

Clinical evidence of congestive heart failure after acute myocardial infarction has been considered by many to be a contraindication to $\beta$ adrenergic blockade and was an exclusion criterion in the major relevant trials. ${ }^{10} 11$ It is estimated that even in the post-thrombolytic era some $32 \%$ of postmyocardial infarction patients have clinical evidence of heart failure, requiring treatment with a diuretic. ${ }^{12}$ Therefore it is not surprising that evidence of heart failure and use of diuretic agents are the most frequent reasons for not prescribing $\beta$ lockers after myocardial infarction. ${ }^{7}$

The use of $\beta$ blockers in survivors of myocardial infarction with evidence of congestive heart failure represents a therapeutic paradox for the clinician. Although $\beta$ blockers are seen as "cardioprotective" in that they reduce mortality and prevent reinfarction, they appear to be contraindicated in this high risk group of patients. Yet left ventricular dysfunction is the strongest single predictor of prognosis after myocardial infarction, so any treatment that can further reduce mortality in this group of patients would be of particular value. ${ }^{13}$

Results from randomised trials specifically designed to test the role of $\beta$ blockade in patients with heart failure after myocardial infarction are not currently available nor are they likely to be for some years.

We therefore conducted a retrospective analysis of optionally prescribed $\beta$ blocker treatment in the AIRE study, in which patients with clinical evidence of congestive heart failure after myocardial infarction were randomly allocated to receive placebo or the ACE inhibitor ramipril. We wished to examine the mortality and morbidity outcomes and modes of death with respect to $\beta$ blocker use and relate these to evidence of impaired ventricular 
function. In contrast to all previous retrospectively analysed studies, the AIRE study population was comprised exclusively of patients with prospectively defined and identified clinical evidence of heart failure after myocardial infarction. Therefore an analysis of the clinical outcomes associated with the empirical use of $\beta$ blockers in this population was considered to be of both clinical and scientific interest.

\section{Methods}

PATIENTS

The design, outcome definitions, and results of the AIRE study have been published elsewhere. ${ }^{14-16}$ Briefly, this was a multinational, randomised, double blind, placebo controlled, parallel group study. Patients were eligible for inclusion if they had a definite acute myocardial infarction and clinical evidence of transient or persistent heart failure (S3 gallop and/or radiographic and/or clinical evidence of pulmonary venous congestion) at any time from hospital admission to randomisation. Exclusion criteria were recognised contraindications to ACE inhibitor treatment, severe heart failure (usually New York Heart Association (NYHA) class IV), heart failure of primary valvar or congenital aetiology, and unstable angina. Overall, 1014 patients were randomised to ramipril and 992 to placebo, starting from day 2 to day 9 after the index myocardial infarct (day 0). Follow up was for a minimum of six months and an average of 15 months.

Decisions as to whether to prescribe $\beta$ blocker or diuretic treatment (as with all other than trial ramipril or placebo treatments) were made by attending physicians, based on clinical judgment. It was expected that the intensity and persistence of clinical evidence of left ventricular dysfunction-along with other factors such as local medical practice-would have affected decision making. To minimise bias, comprehensive multivariate analysis methods were applied to examine associations between

Table 1 Patient characteristics and treatments according to diuretic use at randomisation

\begin{tabular}{llll}
\hline Variable & $\begin{array}{l}\text { Not on diuretic } \\
(n=798)\end{array}$ & $\begin{array}{l}\text { On diuretic } \\
(n=1188)\end{array}$ & $p$ Value \\
\hline Mean (SD) age (years) & $62.8(11.4)$ & $66.6(10.0)$ & $<0.001$ \\
Digitalis & $48(6.0)$ & $195(16.4)$ & $<0.001$ \\
$\beta$ Blocker & $213(26.7)$ & $230(19.4)$ & $<0.001$ \\
Thrombolysis & $500(62.7)$ & $642(54.0)$ & $<0.001$ \\
Nitrates & $412(62.7)$ & $697(58.7)$ & 0.002 \\
Multiple clinical signs of heart failure & $375(47.5)$ & $634(53.5)$ & 0.01 \\
$\quad$ Radiographic & $393(49.7)$ & $635(53.6)$ & 0.09 \\
$\quad$ Rales & $661(83.6)$ & $1043(88.1)$ & 0.005 \\
$\quad$ S3 gallop & $189(23.9)$ & $293(24.7)$ & 0.66 \\
Past medical history & $155(19.4)$ & $293(24.7)$ & 0.006 \\
$\quad$ Myocardial infarction & $53(6.6)$ & $110(9.3)$ & 0.04 \\
$\quad$ Heart failure & $41(5.2)$ & $81(6.8)$ & 0.13 \\
Arrhythmia & $268(33.6)$ & $440(37.0)$ & 0.13 \\
$\quad$ Angina & $220(27.6)$ & $334(28.1)$ & 0.79 \\
$\quad$ Hypertension & $95(11.9)$ & $145(12.2)$ & 0.84 \\
$\quad$ Diabetes mellitus & $608(76.2)$ & $853(71.8)$ & 0.03 \\
Sex (male) & $38(14.3)$ & $40(15.0)$ & 0.09 \\
Mean (SD) LVEF (\%) & $418(52.4)$ & $586(49.3)$ & 0.19 \\
Ramipril & $628(78.7)$ & $915(77.0)$ & 0.38 \\
Aspirin & $121(15.0)$ & $196(16.5)$ & 0.43 \\
Calcium antagonists & & & \\
Location of infarct & $462(57.9)$ & $689(58.0)$ & 0.96 \\
$\quad$ Anterior & $305(38.2)$ & $434(36.5)$ & 0.45 \\
Inferior & $491(63.8)$ & $715(63.2)$ & 0.81 \\
ECG classification: Q wave & & & \\
\hline
\end{tabular}

Values in parentheses are percentages unless otherwise stated.

LVEF, left ventricular ejection fraction. $\beta$ blocker treatment at randomisation and subsequent clinical outcomes.

Diuretic treatment at randomisation was used as an indicator of either transient $/$ mild (low risk) or persistent/more severe (high risk) heart failure. Postmyocardial infarction patients requiring diuretic treatment have more severe clinical heart failure, larger infarcts, and lower left ventricular ejection fractions, and hence are at greater risk of death. ${ }^{17}$ Moreover, the need for diuretic treatment in an already high risk population with clinical evidence of heart failure is associated with a further twofold increase in early mortality. ${ }^{18}$ Retrospective data from other studies suggest that high risk postinfarction patients have the most to gain in absolute terms from $\beta$ blockade. Therefore to test this concept the use of $\beta$ blockade was examined separately in high risk/persistent heart failure and low risk/transient heart failure groups.

END POINTS

We studied the association between $\beta$ blocker use and total mortality, severe/resistant heart failure occurrence, and mode of death during the entire study follow up:

Severe/resistant heart failure (SRHF) was prospectively defined as clinical judgment of severe heart failure (usually NYHA class IV) that is, unresponsive to non-ACE-inhibitor treatment. Onset was usually the date at which open label ACE inhibitor treatment was started.

Details of the definitions and classification of mode of death in the AIRE study have been described elsewhere. ${ }^{16}$ Two modes of death were examined in the current analysis. Sudden cardiac death included sudden collapse, death from an identified arrhythmia, cardiac arrest in the absence of pre-existing circulatory failure, and unwitnessed deaths. Death from circulatory failure included shock (that is, hypotension insufficient to maintain clinically adequate cerebral perfusion for more than 15 minutes before cessation of cardiac activity) and pulmonary oedema. In addition, two routes to death were defined, depending on whether SRHF preceded death.

\section{STATISTICAL ANALYSIS}

Baseline characteristics of different patient groups were compared by use of two sample $t$ test for continuous variables and $\chi^{2}$ tests for categorical variables.

Hazard ratios, 95\% confidence intervals (CI), and $\mathrm{p}$ values of the tested variables were derived from univariate and multivariate Cox proportional hazard analyses. To minimise any confounding, all available baseline variables were simultaneously ${ }^{19}$ entered into a Cox proportional hazards multivariate analysis $(\beta$ blocker, diuretic, digoxin, nitrate, aspirin, and calcium antagonist treatments at randomisation; allocation to ramipril; thrombolytic treatment for the index myocardial infarction; presence of inspiratory rales, S3 gallop and radiographic evidence of pulmonary venous congestion at any time from hospital admission to randomisation; left ventricular ejection 
Table 2 Mortality and severe/resistant heart failure (SRHF) outcomes by baseline clinical features in 1986 patients

\begin{tabular}{lll}
\hline & $\begin{array}{l}\text { Total mortality hazard } \\
\text { ratios }(95 \% \text { CI })\end{array}$ & $\begin{array}{l}\text { SRHF hazard ratios } \\
\text { (95\% CI) }\end{array}$ \\
\hline Past medical history of heart failure & $1.76(1.31$ to 2.38$)$ & $2.17(1.57$ to 3.01$)$ \\
p Value & $<0.001$ & $<0.001$ \\
Past medical history of diabetes mellitus & $1.60(1.23$ to 2.10$)$ & $1.25(0.92$ to 1.71$)$ \\
p Value & $<0.001$ & 0.16 \\
S3 gallop & $1.46(1.16$ to 1.85$)$ & $1.72(1.33$ to 2.22$)$ \\
p Value & 0.016 & $<0.001$ \\
Left ventricular ejection fraction $<39 \%$ & $1.42(0.93$ to 2.18$)$ & $1.41(0.90$ to 2.23$)$ \\
p Value & 0.10 & 0.14 \\
Digoxin & $1.41(1.07$ to 1.86$)$ & $1.19(0.87$ to 1.63$)$ \\
p Value & 0.014 & 0.28 \\
Diuretics & $1.37(1.08$ to 1.73$)$ & $1.50(1.16$ to 1.96$)$ \\
p Value & 0.009 & 0.024 \\
Past medical history of angina & $1.34(1.06$ to 1.68$)$ & $1.18(0.91$ to 1.53$)$ \\
p Value & 0.013 & 0.22 \\
Anterior myocardial infarction & $1.28(1.03$ to 1.61$)$ & $1.11(0.35$ to 3.53$)$ \\
p Value & 0.029 & 0.83 \\
Radiographic evidence of heart failure & $1.22(0.98$ to 1.51$)$ & $1.27(1.00$ to 1.62$)$ \\
p Value & 0.078 & 0.048 \\
Past medical history of myocardial infarction & $1.08(0.85$ to 1.39$)$ & $1.46(1.12$ to 1.91$)$ \\
p Value & 0.55 & 0.005 \\
Age (per 1 year) & $1.03(1.02$ to 1.04$)$ & $1.02(1.01$ to 1.04$)$ \\
p Value & $<0.001$ & $<0.001$ \\
Ramipril & $0.80(0.65$ to 0.99$)$ & $0.82(0.65$ to 1.04$)$ \\
p Value & 0.041 & 0.09 \\
Thrombolysis & $0.68(0.55$ to 0.85$)$ & $0.75(0.59$ to 0.96$)$ \\
p Value & $<0.001$ & 0.02 \\
$\beta$ Blockers & $0.66(0.48$ to 0.90$)$ & $0.58(0.40$ to 0.83$)$ \\
p Value & 0.008 & 0.003 \\
\hline
\end{tabular}

Hazard ratios derived by multivariate analysis with either total mortality or severe/resistant heart failure as an end point in the entire AIRE study population. Covariates were included in this table if $\mathrm{p} \leqslant 0.1$ for at least one analysis and were ordered by size of hazard ratio for total mortality analysis.

fraction; age; sex; previous medical history of heart failure, myocardial infarction, diabetes mellitus, angina, and hypertension; ECG classification of the index myocardial infarction as to its location and $\mathrm{Q}$ wave development). Measurements of left ventricular ejection fraction using multiple methods were available in a cohort of 557 patients $(28 \%)$ and were entered into the analysis as a categorical variable using the mean $(39 \%)$ as a cut off point, with a separate value for non-available measurements.

The same multivariate Cox proportional hazard model was applied to examine sub-

Table 3 Baseline characteristics and treatments according to $\beta$ blocker use at randomisation

\begin{tabular}{llll}
\hline & $\begin{array}{l}\text { Not on } \beta \text { blocker } \\
(n=1543)\end{array}$ & $\begin{array}{l}\text { On } \beta \text { blocker } \\
(n=443)\end{array}$ & $p$ Value \\
\hline Mean (SD) age (years) & $65.7(10.6)$ & $63.1(11.1)$ & $<0.001$ \\
Diuretic & $958(62.1)$ & $230(51.9)$ & $<0.001$ \\
Multiple clinical signs of HF & $827(53.6)$ & $182(41.1)$ & $<0.001$ \\
$\quad$ Radiographic & $829(53.7)$ & $199(44.9)$ & 0.001 \\
Rales & $1345(87.2)$ & $359(81.0)$ & 0.002 \\
S3 gallop & $394(25.5)$ & $88(19.9)$ & 0.016 \\
Digitalis & $213(13.8)$ & $30(6.8)$ & $<0.001$ \\
Calcium antagonist & $273(17.7)$ & $44(9.9)$ & $<0.001$ \\
Aspirin & $1166(75.6)$ & $377(85.1)$ & $<0.001$ \\
Mean (SD) LVEF (\%) & $38.5(14.8)$ & $40.3(13.7)$ & 0.17 \\
Location of infarct & & & \\
Anterior & $870(54.6)$ & $281(63.4)$ & 0.008 \\
Inferior & $587(38.0)$ & $152(34.3)$ & 0.15 \\
Thrombolysis & $863(55.9)$ & $279(63.0)$ & 0.009 \\
Past medical history & & & \\
Angina & $565(36.2)$ & $143(32.3)$ & 0.12 \\
Arrhythmia & $101(6.6)$ & $21(4.8)$ & 0.17 \\
Heart failure & $132(8.5)$ & $31(7.0)$ & 0.29 \\
Diabetes mellitus & $190(12.3)$ & $50(11.3)$ & 0.56 \\
Myocardial infarction & $352(22.8)$ & $96(21.7)$ & 0.61 \\
Hypertension & $427(27.7)$ & $127(28.7)$ & 0.68 \\
ECG classification: Q wave & $942(61.0)$ & $264(59.6)$ & 0.18 \\
Ramipril & $768(49.8)$ & $236(53.3)$ & 0.20 \\
Sex (male) & $1126(73.0)$ & $335(75.6)$ & 0.25 \\
Nitrates & $856(55.5)$ & $253(57.1)$ & 0.54 \\
\hline N & & & \\
\hline
\end{tabular}

Numbers in parentheses are percentages unless otherwise stated.

$\mathrm{HF}$, heart failure; LVEF, left ventricular ejection fraction. groups of patients who were either receiving (persistent heart failure) or not receiving (transient heart failure) diuretic treatment at the time of randomisation.

All $\mathrm{p}$ values are two tailed, and a significance level of 0.05 was used. Statistical analyses were performed using the SPSS version 6.1.

\section{Results}

DIURETIC USE AND ASSOCIATIONS WITH CLINICAL OUTCOMES

Analysis of the clinical characteristics and concomitant treatments at baseline showed that patients requiring diuretics at randomisation were significantly older and were more likely to be female. They more often had a previous medical history of heart failure and myocardial infarction and they were less likely to have received thrombolytic treatment for the index myocardial infarction. They were more likely to have multiple clinical signs of heart failure and to be receiving concomitant digitalis and nitrate treatment. Finally, as expected the use of $\beta$ blockers in these patients was less common (table 1).

In a cohort of 557 patients from the AIRE study, the average left ventricular ejection fraction was 39\% and there was a trend towards a lower left ventricular ejection fraction in those who required diuretic treatment. The need for diuretic treatment after acute myocardial infarction is associated with lower left ventricular ejection fraction. ${ }^{17}$ Presumably, the relation between diuretic use and a lower left ventricular ejection fraction was attenuated in the AIRE study by the fact that all the patients had clinical evidence of left ventricular dysfunction.

Univariate analysis revealed that diuretic use at randomisation was associated with a highly significant increased risk for all cause mortality (hazard ratio $1.7,95 \%$ CI 1.36 to 2.12 , $\mathrm{p}<0.001$ ) and SRHF (hazard ratio 1.83, 95\% CI 1.42 to $2.34, \mathrm{p}<0.001)$. Furthermore, when the use of diuretics was considered along with the use of $\beta$ blockers, the presence of clinical signs of heart failure, the left ventricular ejection fraction, and 16 other baseline clinical characteristics in a multivariate analysis, the need for diuretic treatment at randomisation remained an independent and highly significant predictor of increased mortality and SRHF incidence (table 2). Patients on diuretics at randomisation were more likely to need open label ACE inhibitor treatment during the follow up period $(15.3 \% v 10.9 \%, \mathrm{p}=0.005)$.

Circulatory failure deaths were significantly more common in patients treated with diuretics (hazard ratio $1.54,95 \%$ CI 1.03 to 2.32 , $\mathrm{p}=0.037$ ) and appeared to account for most of the increased total mortality risk in this group.

\section{CLINICAL SIGNS OF HEART FAILURE AND} ASSOCIATIONS WITH CLINICAL OUTCOMES Among the subset of patients in whom the left ventricular ejection fraction was determined, individuals with multiple $(>1)$ clinical signs of heart failure (S3 gallop, inspiratory rales, radiographic evidence of pulmonary venous 


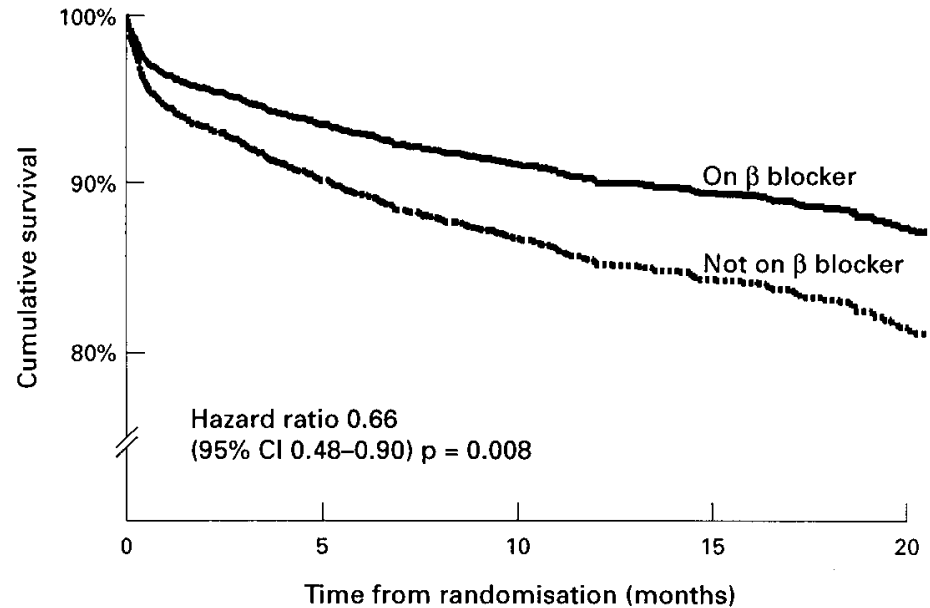

Numbers at risk

$\begin{array}{lrrrrr}\text { On } \beta \text { blocker } & 443 & 407 & 315 & 205 & 76 \\ \text { Not on } \beta \text { blocker } & 1543 & 1334 & 1064 & 729 & 384\end{array}$

Figure 1 Cumulative survival curves according to $\beta$ blocker treatment at randomisation (derived from the multivariate Cox regression analysis). CI, confidence interval.

congestion) had a significantly lower mean left ventricular ejection fraction (mean (SD), 36.6 (13.4)\% v 43 (15.8)\%, p < 0.001).

Univariate analysis showed that the presence of multiple clinical signs of heart failure was a highly significant predictor of the risk of increased mortality (hazard ratio $1.41,95 \%$ CI 1.15 to $1.72, \mathrm{p}=0.009$ ) and SRHF (hazard ratio $1.57,95 \%$ CI 1.25 to $1.97, \mathrm{p}<0.001)$. In the multivariate analysis, $\mathrm{S} 3$ gallop was the only independent heart failure sign predictive of mortality risk and SRHF incidence in the entire study population and in both groups of patients with transient and persistent heart failure (table 2). Radiographic evidence of heart failure was an independent predictor of SRHF occurrence and was associated with a trend towards increased mortality risk in the entire study population. The presence of

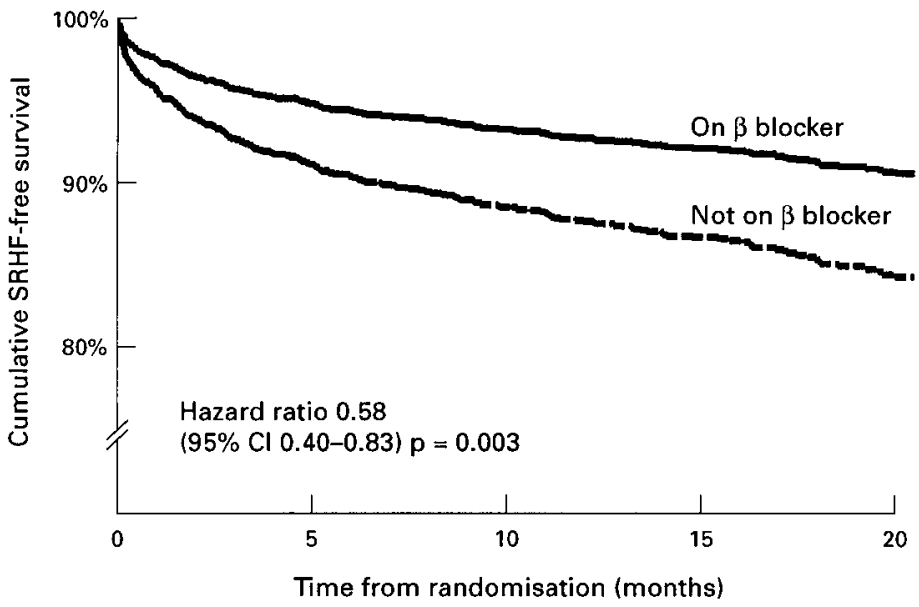

Numbers at risk

$\begin{array}{lrrrrr}\text { On } \beta \text { blocker } & 443 & 409 & 324 & 214 & 77 \\ \text { Not on } \beta \text { blocker } & 1543 & 1359 & 1105 & 758 & 402\end{array}$

Figure 2 Cumulative severe/resistant heart failure (SRHF)-free survival according to $\beta$ blocker treatment at randomisation (derived from the multivariate Cox regression analysis). CI, confidence interval. inspiratory rales did not appear to predict either of these outcomes in any of the groups.

These data suggest that the inclusion of the clinical correlates of left ventricular function into multivariate analysis permits baseline risk adjustment and stratification over and above that denoted by diuretic use, left ventricular ejection fraction, and all the other variables used.

$\beta$ BLOCKER USE AND BASELINE CHARACTERISTICS Analysis of the clinical characteristics and concomitant treatments at baseline revealed that the 443 patients receiving $\beta$ blockers were significantly younger and more likely to have received thrombolytic treatment. Not surprisingly, $\beta$ blockers were prescribed less often when there was evidence of pronounced left ventricular dysfunction with multiple clinical signs of heart failure or the need for diuretic treatment. All three clinical criteria of heart failure were significant negative predictors of $\beta$ blocker prescription. Patients on $\beta$ blockers were less likely to be receiving digitalis and calcium antagonists and more likely to be taking aspirin at randomisation (table 3).

In the patients in whom the left ventricular ejection fraction was determined, $\beta$ blocker treatment did not appear to be associated with a significantly greater mean left ventricular ejection fraction.

OUTCOMES WITH RESPECT TO $\beta$ BLOCKER USE Mortality and SRHF incidence analysis

Univariate analysis showed that $\beta$ blocker use was associated with a lower all cause mortality risk (52 deaths $(11.7 \%$ ) in the $\beta$ blocker group, 340 deaths $(22 \%)$ in those not on $\beta$ blocker treatment; hazard ratio $0.53,95 \%$ CI 0.40 to $0.72, p<0.001$ ) and a lower risk of SRHF development (41 events $(9.2 \%)$ in the $\beta$ blocker group, 280 events $(18.1 \%)$ in those not on $\beta$ blocker treatment; hazard ratio $0.49,95 \%$ CI 0.35 to $0.68, p<0.001$ ). Importantly, when $\beta$ blocker treatment was considered along with 21 other covariates in a multivariate analysis, it remained an independent and highly significant predictor of reduced total mortality (hazard ratio $0.66,95 \%$ CI 0.48 to $0.90, p=0.008$ ) and SRHF occurrence (hazard ratio $0.58,95 \%$ CI 0.40 to $0.83, \mathrm{p}=0.003$ ) (table 2 , figs 1 and 2). Though confidence intervals were wide, the reductions in the relative risk associated with the $\beta$ blocker use appeared smaller in the multivariate analysis, indicating that efficacy may have been partially influenced by covariates. However, despite attenuation of the univariate analysis estimate of risk, the association between $\beta$ blocker treatment and improved outcomes was still highly significant and the most sizeable (by hazard ratio ranking) of all the other beneficial postmyocardial infarction treatments.

In keeping with these findings, patients on $\beta$ blockers at randomisation were less likely to need open label ACE inhibitor treatment during the follow up period $(10 \% v 14.6 \%$, $\mathrm{p}=0.012$ ).

To evaluate the soundness of these findings we conducted a series of sensitivity analyses. 


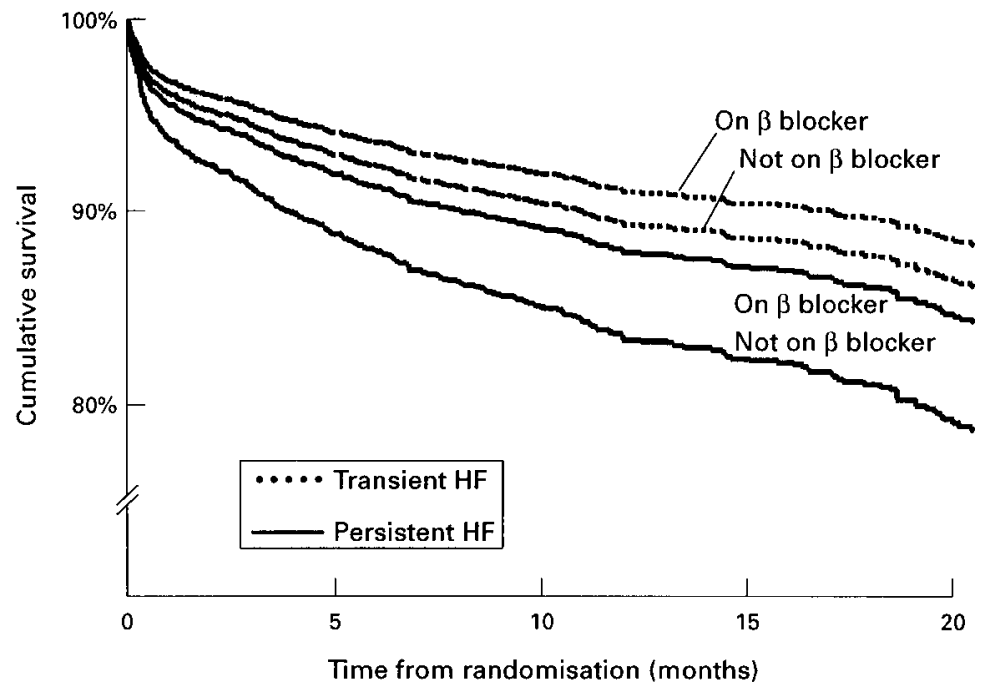

Figure 3 Cumulative survival curves according to $\beta$ blocker treatment in the transient heart failure (HF) group (hazard ratio 0.78, 95\% confidence interval (CI) 0.45 to 1.33, $p=0.36$ ) and the persistent HF group (hazard ratio $0.59,95 \%$ CI 0.40 to 0.86 , $p=0.007$ ) (derived from the multivariate Cox regression analysis).

For a more precise correction for the degree of left ventricular dysfunction, the left ventricular ejection fraction was entered as a continuous variable in our multivariate analysis model. As a result, less than one third of the study population was examined and inevitably the power of the analysis fell considerably. Although no longer statistically significant, the degree of lowering of the relative risks of total mortality (hazard ratio $0.67,95 \%$ CI 0.34 to 1.33 , $\mathrm{p}=0.25$ ) and SRHF (hazard ratio $0.54,95 \%$ CI 0.24 to $1.20, \mathrm{p}=0.12$ ) remained essentially unchanged.

When multivariate analysis of all cause mortality was conducted only in the high risk, persistent heart failure group, $\beta$ blocker treatment was still associated with a significant decrease in risk (hazard ratio $0.59,95 \%$ CI 0.40 to 0.86 , $\mathrm{p}=0.007$ ). In the lower risk, transient heart failure group, $\beta$ blocker treatment was associated with a mortality benefit, though statistical

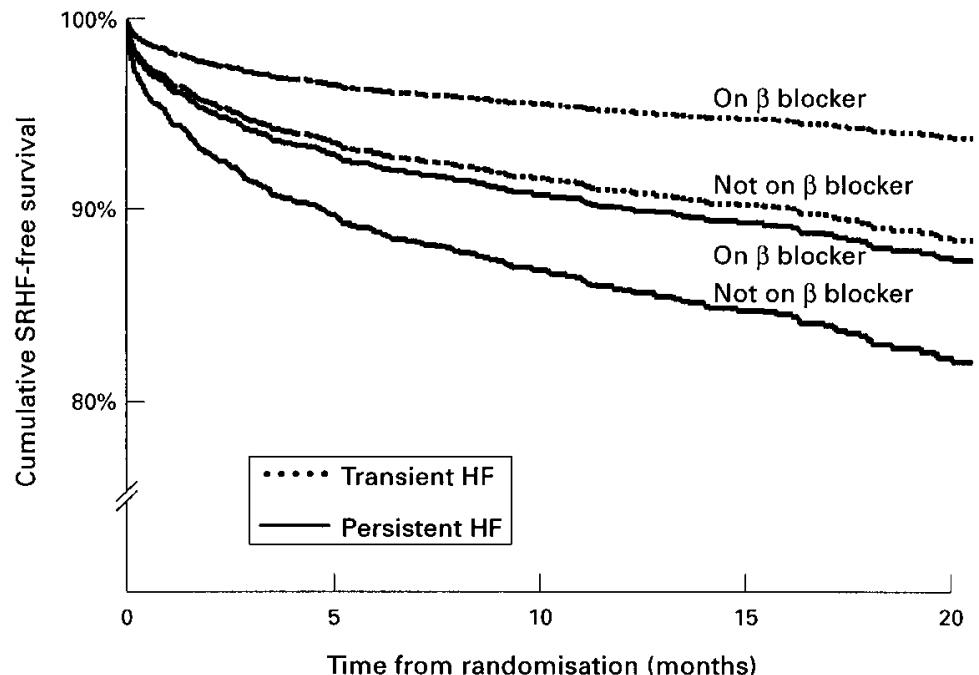

Figure 4 Cumulative severe/resistant heart failure (SRHF)-free survival curves according to $\beta$ blocker treatment in the transient HF group (hazard ratio $0.56,95 \%$ confidence interval (CI) 0.28 to $1.11, p=0.096$ ) and the persistent HF group (hazard ratio $0.58,95 \%$ CI 0.38 to $0.89, p=0.012$ ) (derived from the multivariate Cox regression analysis). significance was not demonstrated (hazard ratio $0.78,95 \%$ CI 0.45 to $1.33, p=0.36$ ) (fig 3). In a similar way, $\beta$ blocker use was associated with a lower SRHF occurrence risk in both the persistent and transient heart failure groups (hazard ratios $0.58,95 \%$ CI 0.38 to $0.89, p=0.012$; and $0.56,95 \%$ CI 0.28 to $1.11, \mathrm{p}=0.096)$, though in the latter group this association was not significant (fig 4).

In the all cause mortality multivariate analysis, all the suspected interactions between variables were tested. The only significant interaction found was that between diuretic treatment and left ventricular ejection fraction $\left(\chi^{2}=10.3\right.$ on 1 degree of freedom, $\mathrm{p}=0.004$ ), indicating that the poor outcome associated with diuretic treatment most probably reflects the lower left ventricular ejection fraction among patients in need of this treatment.

\section{Mode of death analysis}

In the entire AIRE population, multivariate analysis showed that $\beta$ blocker treatment was independently associated with a significantly lower risk of sudden death (hazard ratio 0.61, $95 \%$ CI 0.40 to $0.93, p=0.02$ ) and death not preceded by SRHF (hazard ratio $0.61,95 \% \mathrm{CI}$ 0.37 to $1.00, p=0.047)$. A trend towards lower risk of death from circulatory failure (hazard ratio $0.65,95 \%$ CI 0.38 to $1.09, \mathrm{p}=0.1$ ) and death preceded by SRHF (hazard ratio 0.69, $95 \%$ CI 0.46 to $1.03, p=0.066$ ) was also observed in patients treated with $\beta$ blockers.

\section{Discussion}

This retrospective analysis of the optional use of $\beta$ blocker treatment in the AIRE study indicates that $\beta$ blockade in patients with acute myocardial infarction and clinical evidence of heart failure is associated with improved outcomes. However, as patients were not randomly allocated to $\beta$ blocker treatment, the results should be treated cautiously. To correct for lack of randomisation we applied the principles of covariate adjustment ${ }^{20}$ to account for differences in all available clinical and historical variables. Further supporting the validity of these findings is their consistency with the data from other postmyocardial infarction trials, though again these were not prospectively designed to address this issue.

The large number of events in the AIRE study (392 deaths and 321 SRHF occurrences) means that suggested criteria for overfitting data were not violated. All the outcomes associated with $\beta$ blocker treatment were evaluated for adherence to the assumption of proportional hazards and in all cases the criteria of proportionality were fulfilled.

As discussed previously, the use of $\beta$ blocker treatment in the AIRE study is anticipated to have been heavily biased by the clinical and laboratory estimation of the degree of the left ventricular dysfunction. Therefore the need for diuretic treatment and the clinical signs of heart failure present at any time before randomisation served as clinical correlates of left ventricular function, ${ }^{17}{ }^{21-25}$ permitting the necessary adjustments. The importance of simple clinical criteria is emphasised by the fact 
that for any given level of ejection fraction clinical evidence of heart failure is still an independent prognostic predictor, which more than doubles mortality in a postinfarct population. ${ }^{23}{ }^{24}$ Furthermore, information on left ventricular ejection fraction was available for an appreciable number of patients, and was accordingly used in the multivariate analysis, allowing additional adjustment.

Clinical outcomes with respect to $\beta$ blocker treatment were examined separately in high and lower risk groups of patients, using identical methods. These groups were defined according to whether patients were receiving diuretic treatment at baseline. Multivariate analysis showed $\beta$ blocker treatment to be a significant independent predictor of decreased risk of all cause mortality only in the high risk group of patients. In the lower risk patients with transient evidence of heart failure, $\beta$ blocker treatment was not a significant independent predictor of total mortality risk, probably as a result of the lower number of deaths observed. However, when the response to $\beta$ blockade in the two subgroups was compared formally, there was no statistically significant difference between them. Similar trends were observed in the multivariate analysis of the SRHF occurrence with respect to $\beta$ blocker treatment. Once more, $\beta$ blocker treatment at baseline was an independent predictor of reduced progress to $\mathrm{SRHF}$ in the group with persistent heart failure.

It appears that when $\beta$ blocker treatment was used in a high risk patient, the mortality and SRHF risks were significantly reduced, being similar to those of lower risk individuals.

Among the clinical signs of heart failure, the presence of S3 gallop was the strongest predictor of increased total mortality, SRHF occurrence, and failure to prescribe $\beta$ blockers. At the other end of the spectrum, the presence of inspiratory crackles did not appear to predict any of the clinical outcomes examined but was predictive of failure to prescribe $\beta$ blockers.

Concerning mode of death, we observed that $\beta$ blocker treatment was associated with a significantly lower risk of sudden death in the AIRE study population. The route to death without preceding SRHF was also less common in the $\beta$ blocker treated patients. These findings are in keeping with the results of other studies showing $\beta$ blockade to be particularly effective in reducing sudden death in high risk patients with evidence of heart failure. ${ }^{25-27}$ Moreover, $\beta$ blocker treatment was also associated with a trend towards lower risk of death from circulatory failure and death preceded by SRHF, alleviating fears to the contrary in patients with clinical evidence of heart failure after myocardial infarction.

DATA FROM OTHER STUDIES

A few of the large randomised, postmyocardial infarction, $\beta$ blocker trials conducted before the routine use of thrombolytic and ACE inhibitor treatment did include small numbers of patients with compensated mild to moderate heart failure. ${ }^{25}$ 28-32 Although they were not designed to test the role of $\beta$ blockade in patients with heart failure, subgroup analysis of these trials supports the policy of a more liberal use of $\beta$ blocker treatment in patients with evidence of mild to moderate left ventricular dysfunction. A recent survey of all the trials which provided mortality data for subsets of patients with left ventricular dysfunction indicates that $\beta$ blocker treatment was well tolerated and was associated with a $20-30 \%$ risk reduction in total mortality. ${ }^{33}$ Noticeably, this was the same mortality reduction as was observed in the entire population. Because of the high mortality among patients with left ventricular dysfunction, the absolute gain in numbers of lives saved per 100 patients treated with $\beta$ blockers is much larger than that in patients with preserved ventricular function. Our findings are consistent with data suggesting that $\beta$ blocker treatment after myocardial infarction is particularly beneficial in patients with evidence of left ventricular dysfunction and other groups at higher risk. ${ }^{325}$ 28-30 32-35

Lichstein et al carried out a retrospective analysis of non-randomised $\beta$ blocker use in the multicenter diltiazem post-infarction trial $(M D P I T)^{36}$ and found that in various left ventricular ejection fraction and heart failure strata, $\beta$ blocker treatment appeared to be a predictor of decreased all cause mortality and increased heart failure-free survival. However, it is unknown whether $\beta$ blocker treatment would have remained a significant independent predictor of mortality had a multivariate analysis model adjusting for all these indicators of left ventricular function been performed. Similarly, Kennedy et al analysed the optional, nonrandomised use of $\beta$ blockers in the cardiac arrhythmia suppression trial (CAST) ${ }^{27}$ They included 2611 patients with a left ventricular ejection fraction of $\leqslant 40 \%$ but only $16 \%$ had a history of congestive heart failure. Multivariate analysis using a Cox regression model including 12 baseline historical and clinical characteristics showed that $\beta$ blocker treatment was associated with a decrease in all cause mortality risk.

Evaluation of $\beta$ blocker use in a different population from our own, in an ACE inhibition postmyocardial infarction trial (survival and ventricular enlargement study) selecting asymptomatic patients with a radionuclide left ventricular ejection fraction of $\leqslant 40 \%$ has recently been reported. ${ }^{37}$ Multivariate analysis correcting for nine baseline clinical characteristics showed that $\beta$ blocker treatment at randomisation was independently associated with a lower cardiovascular mortality and occurrence of severe heart failure.

In contrast to each of the above studies, all patients in the AIRE study had clinical evidence of heart failure after myocardial infarction. In addition, the AIRE study database allowed us to address the issue of postmyocardial infarction $\beta$ blocker treatment in the modern context, where aspirin, thrombolytic, and ACE inhibitor treatments are routinely used. Of particular interest is our finding that the beneficial effects of $\beta$ blockers and ACE inhibitors were independent and multiplicative, both effects appearing more marked 
in patients requiring concomitant diuretic treatment.

B ADRENERGIC RECEPTOR BLOCKADE IN CHRONIC HEART FAILURE AND AFTER MYOCARDIAL

INFARCTION

To date, a growing body of evidence indicates that $\beta$ blockers may have a role in the treatment of patients with congestive heart failure. ${ }^{38-41}$ Three large mortality studies of $\beta$ blockade in chronic heart failure are under way at present and are expected to provide a definitive answer (MERIT, BEST, and CIBIS-II). ${ }^{42-44}$

Although uncertainty persists, the beneficial actions of $\beta$ blockers in this setting may result from antagonism of the activated neurohumoral axis, upregulation of the $\beta$ adrenoceptors, and anti-ischaemic, antiarrhythmic, and favourable haemodynamic effects. ${ }^{39456}$ Most of these actions would be particularly welcome when left ventricular dysfunction develops after myocardial infarction. ${ }^{47}$

Whether clinical evidence of heart failure following myocardial infarction is caused by a rise in end diastolic pressure associated with ischaemia rather than "true" myocardial necrosis is of little importance, as the value of $\beta$ blockade in preventing the evolution of unstable angina to myocardial infarction ${ }^{48}$ and in reducing infarct size $^{49}$ and reinfarction rate $^{2}$ have all been shown convincingly. $\beta$ Blockade should be particularly effective in eliminating any component of reversible ischaemia in this setting.

In conclusion, optional $\beta$ blocker treatment after myocardial infarction in patients with clinical evidence of heart failure was associated with an independent decrease in total mortality and progression to severe heart failure. Importantly, this association remained statistically significant and substantial in the high risk group of patients who required diuretic treatment and had more marked and persistent heart failure. This is the very group of patients in whom the use of $\beta$ blockers has generally been avoided by clinicians.

These results reinforce accumulating data that suggest that high risk patients with evidence of heart failure following myocardial infarction derive at least the same relative, and therefore greater absolute, benefit from $\beta$ blockade as low risk patients without evidence of heart failure. Treating such patientsparticularly those with more severe, overt heart failure-will require considerable care. Large scale randomised trials are needed urgently to confirm or refute these findings and establish appropriate clinical practice.

1 The Task Force on the Management of Acute Myocardial Infarction of the European Society of Cardiology. Acute myocardial infarction: pre-hospital and in-hospital management. Eur Heart F 1996;17:43-63.

2 Yusuf S, Peto R, Lewis J, et al. Beta blockade during and after myocardial infarction: an overview of the randomized trials. Prog Cardiovasc Dis 1985;27:335-71.

3 The Beta-Blocker Pooling Project Research Group. The Beta-Blocker Pooling Project Research Group (BBPP): subgroup findings from randomized trials in postinfarction patients. Eur Heart f 1988;9:8-16.

4 Kendall MJ, Lynch KP, Hjalmarson A, et al. $\beta$-Blockers and sudden cardiac death. Ann Intern Med 1995;123:358-67.

5 Yusuf S, Lessem J, Jha P, et al. Primary and secondary prevention of myocardial infarction and strokes: an update of vention of myocardial infarction and strokes: an update of 11: $61-73$.
6 Ball SG. Beta-blockers and antithrombotics for secondary prevention after myocardial infarction. Eur Heart 7 1998;19:14-15.

7 Viskin S, Kitzis I, Lev E, et al. Treatment with betaadrenergic blocking agents after myocardial infarction: from randomized trials to clinical practice. $\mathcal{F} \mathrm{Am}$ Coll Cardiol 1995;25:1327-32.

8 Brand DA, Newcomer LN, Freiburger A, et al. Cardiologists' practices compared with guidelines: use of $\beta$-blockade after acute
Cardiol 1995;26:1432-6.

9 Lamas GA, Pfeffer MA, Hamm P, et al, for the SAVE Investigators. Do the results of randomized clinical trials of cardiovascular drugs influence medical practice? $N$ Engl $\mathcal{F}$ Med 1992;327:241-7.

10 Julian DG. Can $\beta$-blockers be safely used in patients with recent myocardial infarction who also have congestive heart failure? Circulation 1983;67:I91.

11 Shand DG. Clinical pharmacology of the beta blocking drugs: implications for the post infarct patient. Circulation 1983;67:I2-5.

12 Stevenson R, Radjadaylan K, Wilkinson P, et al. Short and long term prognosis of acute myocardial infarction since introduction of thrombolysis. BMF 1993;307:349-53.

13 Moss AJ, Bigger JT, Odoroff CL. Postinfarct risk stratification. Prog Cardiovasc Dis 1987;29:389-412.

14 The Acute Infarction Ramipril Efficacy (AIRE) Study Investigators. Effect of ramipril on mortality and morbidity of survivors of acute myocardial infarction with clinical evidence of heart failure. Lancet 1993;342:821-8.

15 Hall AS, Winter C, Bogle SM, et al, on behalf of the AIRE Study Investigators. The acute infarction ramipril efficacy (AIRE) study: rationale, design, organization, and outcome definitions. $\mathcal{f}$ Cardiovasc Pharmacol 1991;18:105-9.

16 Cleland JGF, Erhardt L, Hall AS, et al, on behalf of the AIRE Study Investigators. Validation of primary and among patients with clinical evidence of heart failure after among patients with clinical evidence of heart failure after a myocardial infarction: a report from the AIRE

17 Vaughan DE, Lamas GA, Pfeffer MA. Role of left ventricular dysfunction in selective neurohumoral activation in the recovery phase of anterior wall acute myocardial infarction. Am f Cardiol 1990;66:529-32.

18 Stubbs DF. Positive synergism between diuretics and methylprednisolone following acute myocardial infarction. F Int Med Res 1986;14:21-4.

19 Simon R, Altman DG. Statistical aspects of prognostic factor studies in oncology. Br F Cancer 1994;69:979-85.

20 Concato J, Feinstein AR, Holford TR. The risks of determining risk with multivariable models. Ann Intern Med 1993;118:201-10.

21 Ahnve S, Gilpin E, Henning $\mathrm{H}$, et al. Limitations and advantages of the ejection fraction for defining high risk after acute myocardial infarction. Am f Cardiol 1986;58: $872-8$.

22 The Multicenter Postinfarction Research group. Risk stratification and survival after acute myocardial infarction. $N$ Engl F Med 1983;329:331-6.

23 Gottlieb S, Moss AJ, McDermott M, et al. Interrelation of left ventricular ejection fraction, pulmonary congestion and outcome in acute myocardial infarction. $A m$ f Cardiol 1992;69:977-84

24 Nicod P, Gilpin E, Dittrich H, et al. Influence on prognosis and morbidity of left ventricular ejection fraction with and without signs of left ventricular failure after acute myocardial infarction. Am $\mathcal{F}$ Cardiol 1988;61:1165-71.

25 Chadda K, Goldstein S, Byington R, et al. Effect of propranolol after acute myocardial infarction in patients propranolol after acute myocardial infarction in patients

26 Szabo BM, Crijns HJGM, Wiesfeld ACP, et al. Predictors of mortality in patients with sustained ventricular tachycardias or ventricular fibrillation and depressed left ventricular function: importance of $\beta$-blockade. Am Heart f 1995;130: $281-6$.

27 Kennedy HL, Brooks MM, Barker AH, et al, for the CAST Investigators. Beta-blocker therapy in the cardiac arrhythmia suppression trial. Am f Cardiol 1994;74:674-80.

28 Gundersen T. Influence of heart size on mortality and reinfarction in patients treated with timolol after myocardial infarction. Br Heart 7 1983;50:135-9.

29 Boissel J-P, Leizorovicz A, Picolet $\mathrm{H}$, et al, for the APSI Investigators. Secondary prevention after high risk acute myocardial infarction with low-dose acebutolol. Am f Cardiol 1990;66:251-60

30 The MIAMI Trial Research Group. Metoprolol in acute myocardial infarction (MIAMI). A randomised placebocontrolled international trial. Eur Heart f 1985; 6: 199-226.

31 The Norwegian Multicenter Study Group. Timololinduced reduction in mortality and reinfarction in patients surviving acute myocardial infarction. $N$ Engl f Med 1981; 304:801-7.

32 Herlitz J, Waagstein F, Lindqvist J, et al. Effect of metoprool on the prognosis for patients with suspected acute myocardial infarction and indirect signs of congestive heart failure. (A subgroup analysis of the Goteborg metoprolol trial.) Am $\mathcal{f}$ Cardiol 1997;80:40-44J.

33 Held P. Effects of beta blockers on ventricular dysfunction after myocardial infarction: tolerability and survival effects. Am f Cardiol 1993;71:39-44C.

34 Furberg CD, Hawkins M, Lichstein E, for the BHAT Study Group. Effect of propranolol in postinfarction patients with mechanical or electrical complications. Circulation 1984; 69:761-5. 
35 Viscoli CM, Horwitz RI, Singer BH. Beta-blockers after myocardial infarction: influence of first-year clinical course 105.
on long-

36 Lichstein E, Hager WD, Gregory JJ, et al, for the Multicenter Diltiazem Post-Infarction Research Group. Relation between $\beta$-adrenergic blocker use, various correlates of left ventricular function and the chance of developing congestive heart failure. F Am Coll Cardiol 1990;16: 1327-32.

37 Vantrimpont P, Rouleau JL, Wun C-C, et al, for the SAVE Investigators. Additive beneficial effects of $\beta$-blockers to angiotensin-converting enzyme inhibitors in the survival and ventricular enlargement (SAVE) study. $7 \mathrm{Am}$ Coll Cardiol 1997;29:229-36.

38 Packer M, Bristow MR, Cohn JN, et al, for the US Carvedilol Heart Failure Group. The effect of carvedilol on morbidity and mortality in patients with chronic heart failure. N Engl f Med 1996;334:1349-55.

39 Cleland JGF, Bristow MR, Erdmann E, et al. Beta-blocking agents in heart failure. Eur Heart f 1996;17:1629-39.

40 CIBIS Investigators and Committees. A randomized trial of $\beta$-blockade in heart failure. Circulation 1994;90:1765-77. $\beta$-blockade in heart failure. Circulation 1994;90:1765-77.
Waagstein F, Bristow MR, Swedberg K, et al, for the MDC

Wrial Study Group. Beneficial effects of metoprolol in idioTrial Study Group. Beneficial effects of metoprolol in idio-
pathic dilated cardiomyopathy. Lancet 1993;342:1441-6.
42 Rationale, design, and organization of the metoprolol CR/XI randomized tntervention trial in heart failure CR/XL randomized tntervention trial in
(MERIT-HF). Am 7 Cardiol 1997;80:54-8J.

43 The BEST Steering Committee. Design of the $\beta$-blocker evaluation survival trial (BEST). Am f Cardiol 1995;75: $1220-3$

44 McMurray J, Cleland J, Cowley A. Ongoing and planned clinical trials in chronic heart failure and left ventricular dysfunction. Exp Opin Invest Drugs 1995;4:1069-80.

45 Sackner-Bernstein JD, Mancini DM. Rationale for treatment of patients with chronic heart failure with adrenergic blockade. $\mathscr{F} A M A$ 1995;274:1462-7.

46 Bristow MR. Pathophysiologic and pharmacologic rationales for clinical management of chronic heart failure with beta-blocking agents. Am 7 Cardiol 1993;71:12-22C

47 Rouleau JL, de Champlain J, Klein M, et al. Activation of neurohumoral systems in postinfarction left ventricular dysfunction. I Am Coll Cardiol 1993;22:390-8.

48 Lubsen J, Tijssen JGP for the HINT Research Group. Efficacy of nifedipine and metoprolol in early treatment of unstable angina in the coronary care unit: findings from the unstable angina in the coronary care unit: findings from the Holland interuniversity nifedipine $/ \mathrm{m}$
(HINT). Am 7 Cardiol 1987;60:18-25A.

49 Timolol International Collaborative Study Group. Reduction of infarct size with early use of timolol in acute myocardial infarction. $N$ Engl f Med 1984;310:9-15. 